\title{
Simulation of single-molecule trapping in a nanochannel
}

\author{
William Neil Robinson \\ Lloyd M. Davis \\ University of Tennessee Space Institute \\ Center for Laser Applications \\ 411 B. H. Goethert Parkway \\ Tullahoma, Tennessee TN 37388
}

\begin{abstract}
The detection and trapping of single fluorescent molecules in solution within a nanochannel is studied using numerical simulations. As optical forces are insufficient for trapping molecules much smaller than the optical wavelength, a means for sensing a molecule's position along the nanochannel and adjusting electrokinetic motion to compensate diffusion is assessed. Fluorescence excitation is provided by two adjacently focused laser beams containing temporally interleaved laser pulses. Photon detection is time-gated, and the displacement of the molecule from the middle of the two foci alters the count rates collected in the two detection channels. An algorithm for feedback control of the electrokinetic motion in response to the timing of photons, to reposition the molecule back toward the middle for trapping and to rapidly reload the trap after a molecule photobleaches or escapes, is evaluated. While accommodating the limited electrokinetic speed and the finite latency of feedback imposed by experimental hardware, the algorithm is shown to be effective for trapping fastdiffusing single-chromophore molecules within a micron-sized confocal region. Studies show that there is an optimum laser power for which loss of molecules from the trap due to either photobleaching or shot-noise fluctuations is minimized. () 2010 Society of Photo-Optical Instrumentation Engineers. [DOI: 10.1117/1.3477320]
\end{abstract}

Keywords: single molecule; fluorescence; nanochannel; trapping; spectroscopy; simulation; diffusion; photon counting.

Paper 10111RR received Mar. 4, 2010; revised manuscript received Jun. 14, 2010 ; accepted for publication Jun. 23, 2010; published online Aug. 6, 2010.

\section{Introduction}

A single fluorescent molecule may be readily detected in a confocal microscope, but diffusion restricts the residence time of the molecule within the probe volume and hence limits the maximum observation time. This paper presents Monte Carlo simulations of the detection and trapping of a single fluorescent biomolecule confined to a nanochannel by use of electrokinetic motion for the countering of diffusion.

Enderlein first described the use of feedback to compensate for diffusion to enhance observation capabilities for single molecules. ${ }^{1}$ He proposed that confocal microscopy can be used to track a fluorescent molecule across a spatial range in a two-dimensional membrane. While this can also be done with wide-field microscopy, confocal microscopy typically provides improved signal-to-noise ratio (SNR) and allows for observation of subnanosecond timing of fluorescence events by use of a single-photon avalanche diode (SPAD) or photomultiplier (PM) detector and time-correlated single-photon counting. This technique also has the desirable property of being able to measure the fluorescence lifetime, but the SPAD or PM detector provides no direct spatial information. However, a focused laser spot scanning in a circular pattern can be used to determine spatial information and thereby perform tracking of a molecule. The signal given by the detector will

Address correspondence to Lloyd M. Davis, University of Tennessee Space Institute, Center for Laser Applications, Tullahoma, TN 37388. Tel: 931-393-7335; Fax: 931-393-7218; E-mail: Idavis@utsi.edu modulate in time according to the position of the molecule, being more constant when the molecule is near the center of the scanning circle and more intensely modulated as it is displaced from this position. If polar coordinates are used, the intensity of the modulation gives the radial coordinate $r$, and the phase of the modulation provides the angular position $\theta$ (Ref. 1). Feedback can then be used to control a piezoelectric translator to track the molecule so that it remains near the middle of the circular pattern.

The technique has been extended to tracking in three dimensions (3-D) by use of a scanning pattern that is also modulated in the axial direction. Berglund and Mabuchi have tracked individual fluorescent particles by use of a scanning laser focus with single-photon excitation. ${ }^{2}$ Two-photon experiments with similar scanning patterns have been performed by the group of Gratton. ${ }^{3}$ There is clear interest in tracking single molecules, particularly proteins within living cells. Levi and Gratton have done work to this end with various probes, including colloidal gold and quantum dots. ${ }^{4}$

Quantum dots have also been tracked in 3-D using another technique. ${ }^{5,6}$ Collected fluorescence is split at a beamsplitter and imaged onto two sets of two adjacent optical fibers, each connected to a separate SPAD detector. The fibers collect light from four points arranged in a tetrahedron to provide position information in all three spatial dimensions. A piezoelectric translation stage controlled by feedback then provides a means for repositioning a single quantum dot to the middle of

$1083-3668 / 2010 / 15(4) / 045006 / 12 / \$ 25.00$ @ 2010 SPIE 
the four points. This technique has enabled tracking of particles with a diffusion coefficient of $\sim 0.7 \mu \mathrm{m}^{2} \mathrm{~s}^{-1}$.

In addition to tracking, many single-molecule studies would benefit from trapping. For example, it is possible to covalently bind a biomolecule of interest to a dielectric bead, which is held with magnetic ${ }^{7}$ or optical tweezers. ${ }^{8}$ Dielectric beads as small as $20 \mathrm{~nm}$ have been optically trapped, and magnetic beads are available in sizes down to a few hundred nanometers. ' In both cases, the effectiveness of trapping scales with the cube of the size of the bead and a very high magnetic field or laser power would be needed to trap a very small bead or biomolecule. Also, the covalent binding of a biomolecule to a substrate or bead may impair its biological activity or otherwise alter its behavior. Hence for studies of single biomolecules, alternative means of trapping the molecule are of interest.

Cohen and Moerner have developed the anti-Brownian electrophoretic (ABEL) trap, which uses feedback to trap a molecule within a thin fluidic cell with four electrodes to control electrokinetic motion in two dimensions (2-D). ${ }^{10-12}$ They initially used a CCD camera for the 2-D position determination. For faster temporal response and feedback, in later experiments, they employed the circular-scanning focused laser spot technique. Four electrodes provide for the electrokinetic transport of the molecule in two dimensions, with the third dimension being confined by the walls of the fluidic device, which are separated by only $\sim 400 \mathrm{~nm}$.

When a molecule in solution is confined to a thin volume between two planar interfaces for trapping in 2-D, it suffers a high rate of collisions with the surfaces (typically, $>10^{4}$ collisions/s, as indicated by our Monte Carlo simulations). For applications that can tolerate such disturbances, a 1-D nanochannel trap should be equally useful but simpler to implement and control. ${ }^{13}$

There has been increasing interest in single-molecule trapping in solution. ${ }^{14}$ Also, experiments on the confinement and detection of single molecules within nanochannels have been reported as early as $1997 .{ }^{15}$ More recently, there have been rapid developments in the capabilities for fabricating nanochannels in lab-on-a-chip devices for single-biomolecule fluorescence detection applications. ${ }^{16}$ Initial experiments on single-molecule detection with actively controlled electrokinetic transport of the solution within a nanochannel in a device fabricated from fused silica have been reported. ${ }^{13}$ Maximum-likelihood data analysis strategies for sensing the position of a single molecule within a trap have also been described. $^{17}$

This paper discusses Monte Carlo computer simulations of the successive delivery and 1-D trapping of single molecules within a nanochannel. The simulations provide a means for developing and testing algorithms that may be implemented in software within a field-programmable gate array (FPGA), which is a part of the hardware for controlling the electrokinetic voltages of the single-molecule trap. ${ }^{13}$ The simulation also enables the robustness of the trap to be studied under different experimental conditions. The goal is to trap a molecule in a nanochannel at detected photon count rates of $\sim 10^{5} \mathrm{~s}^{-1}$ and to rapidly replace it with a new one following photobleaching or escape.

Monte Carlo simulations have been used for validating experiments and determining feasibility limits since the first re- ports on detection of single-chromophore molecules in solution. ${ }^{18}$ The algorithms in early work simulated the number of detected photons in fixed sequential time intervals and have been used to study efficiency of detection, ${ }^{19}$ two-color coincidence detection limits, ${ }^{20}$ and statistical noise in fluorescence correlation spectroscopy. ${ }^{21}$ An algorithm for efficiently generating the time of arrival of each detected photon by use of variable time intervals for the various physical processes was later developed and used for detailed studies of fluorescence correlation spectroscopy, ${ }^{22}$ including the effects of triplet crossing and saturation. ${ }^{23}$ The Monte Carlo simulations presented in this paper use a similar algorithm with variable time intervals for the excitation and photophysical processes to determine the precise timing of each photon, which must be known to implement trapping.

Section 2 presents details of the numerical modeling from which the principles of the trapping procedure become evident. Section 2.1 explains the two-beam pulse-interleaved excitation scheme and time-gated photon counting, which provide information about molecule position; Sec. 2.2 explains the procedures for modeling Brownian diffusion and timed adjustment of electrokinetic transport of molecules; Sec. 2.3 discusses the photophysical processes that molecules undergo and how these are simulated to determine the precise timing of events; Sec. 2.4 discusses photon detection, including photodetector timing jitter, background, dead-time, and afterpulses, as well as the recording of photon time-stamp data in experimental format; and Sec. 2.5 presents the algorithm used for trapping and the reloading of the trap following loss of signal. Section 3 tabulates parameters and presents figures that summarize the predicted behavior of the trap for different experimental settings. Determination of statistical data from the autocorrelation of the sequence of photons is explained, and autocorrelation functions from simulations are compared with those from previously reported experiments. Section 4 concludes with a summary of the major findings.

\section{Numerical Modeling}

\subsection{Laser Excitation Profile and Time-Gated Photon Collection}

In order to determine the position of the molecule along the nanochannel, a time-varying spatial pattern of laser irradiance is used together with time-gated photon detection. The irradiance pattern is formed by splitting the beam from a modelocked laser into two beams, which are then recombined at a second beamsplitter and focused into the nanochannel at two closely spaced points separated by an adjustable distance. The mode-locked laser delivers picosecond pulses separated by $T=13.2 \mathrm{~ns}$, and one of the two beams is delayed by $T / 2$ $=6.6 \mathrm{~ns}$ so that the excitation pulses at each focal spot alternate in time, with $6.6 \mathrm{~ns}$ between the excitations.

The width and depth of the nanochannel $\left(d_{y}, d_{z}\right.$ $\approx 100 \mathrm{~nm}$ ) are much smaller than the beam waist of each laser spot $\left(\omega_{0}=0.5 \mu \mathrm{m}\right)$, and hence the irradiance is approximately constant through each cross section of the nanochannel. The profiles for each laser spot are assumed to be Gaussian along the length $x$ of the nanochannel, and hence the total irradiance from the series of laser pulses is given by 


$$
I(x, t)=I_{1}(x, t)+I_{2}(x, t),
$$

where

$$
\begin{gathered}
I_{1}(x, t)=\frac{\bar{P}}{\pi \omega_{0}^{2}} \exp \left[-2\left(x-x_{1}\right)^{2} / \omega_{0}^{2}\right] \sum_{k} \delta(t-k T), \\
I_{2}(x, t)=\frac{\bar{P}}{\pi \omega_{0}^{2}} \exp \left[-2\left(x+x_{1}\right)^{2} / \omega_{0}^{2}\right] \sum_{k} \delta(t-k T-T / 2),
\end{gathered}
$$

are the irradiance profiles of the left and right laser foci, and where $\bar{P}$ is the combined mean power of both laser beams, which are equal, $\omega_{0}$ is the beam waist of each beam, $x_{1}$ is one-half of the separation between the two laser foci, and the temporal profile of each picosecond laser pulse is represented as $\delta(t)$. Ideally, the separation $2 x_{1}$ is set to be equal to $\omega_{0}$, as this provides greatest slope of the irradiance from each laser spot at the center of the trap and hence greatest sensitivity for position determination. With this beam separation, the total time-averaged irradiance from the two beams is approximately constant between $x=-x_{1}$ and $x_{1}$, as shown in Fig. 1 . This provides the advantage that the time-averaged excitation of a trapped molecule remains constant as it diffuses within the bounds of the trap.

Photon detection is time-gated into two channels. Each channel counts photons that fall within a 6.6-ns interval that follows one set of excitation pulses at one of the two laser foci. Fluorescence photons generated by each laser focus generally fall into the time channel corresponding to that focus. However, if the fluorescence decay takes longer than $6.6 \mathrm{~ns}$, the released photon will be counted after the next laser pulse, causing the photon to be registered in the incorrect time channel. Such events lead to cross talk and decreased precision in the prediction of the molecule position. Also, the singlephoton timing error of the SPAD detector may cause a photon to be registered in the incorrect time channel. This is modeled by adding a random number with a distribution that approximates the SPAD impulse response function to the arrival time of each detected photon. In the results presented in Sec. 3, this

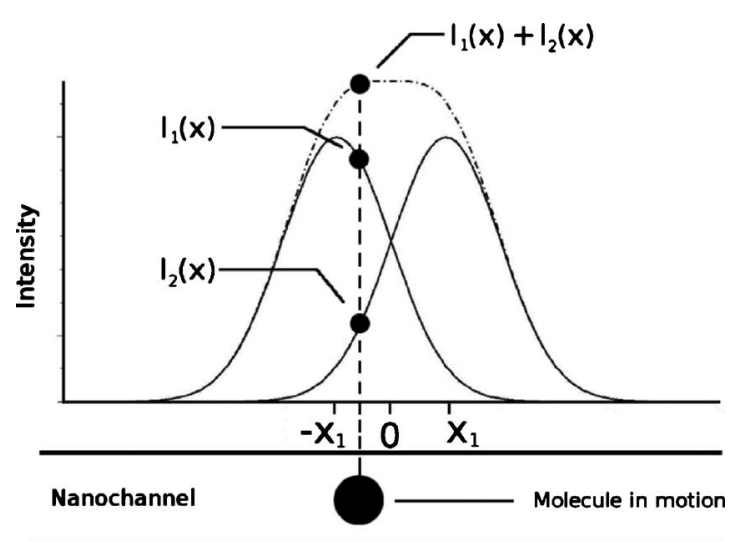

Fig. 1 Irradiance profiles of each of the two laser beams, $I_{1}(x)$ and $I_{2}(x)$, and the total irradiance (dotted-dashed line) experienced by a molecule at a position $x$ within the nanochannel. distribution is taken to be a Gaussian with a standard deviation of $\sigma=127.4 \mathrm{ps}$ and a mean shift of $3 \sigma$, which closely models the experimental distribution. Figure 2 shows the histograms of the time delays between photon detection events and the laser pulses generated by a simulation of trapping in which the fluorescence lifetime is taken to be $\tau_{F}=3.0 \mathrm{~ns}$. In this case, the probability for assignment of a fluorescence photon into the incorrect time channel is $\sim 0.1$. The methods for simulation of background and detector afterpulses are discussed in Sec. 2.4.

\subsection{Molecular Transport}

The numerical simulation considers individual molecules being transported on a one-dimensional grid along the long axis of the nanochannel by Brownian diffusion and also by electrokinetic flow due to the voltage applied across the length of the nanochannel. The grid must be fine compared to the size of the laser waist in order to accurately model the level of excitation of molecules within the focused laser beams. For the results of Sec. 3, the waist is $\omega_{0}=0.5 \mu \mathrm{m}$, and the grid spacing is set to $\Delta x=.01 \mu \mathrm{m}$. The simulation models a nanochannel with a length of $20 \mu \mathrm{m}$, which corresponds to $2 L+1=2001$ grid points.

Electrokinetic flow along the nanochannel is modeled by moving all molecules one grid space in the appropriate direction at appropriate times. If the flow velocity $v_{F}$ is constant, then the moves occur at regular time intervals $\Delta t_{F}$ such that

$$
v_{F} \Delta t_{F}= \pm \Delta x .
$$

However, if the flow is adjusted during trapping, then the time and direction of the next flow step is reevaluated. For example, if the flow velocity is changed from $v_{F}$ to $v_{F}^{\prime}$, at a time $t^{\prime}$ following the last flow step, then the time until the next flow step is $\Delta t_{F}^{\prime}$ such that

$$
v_{F} t^{\prime}+v_{F}^{\prime}\left(\Delta t_{F}^{\prime}-t^{\prime}\right)= \pm \Delta x .
$$

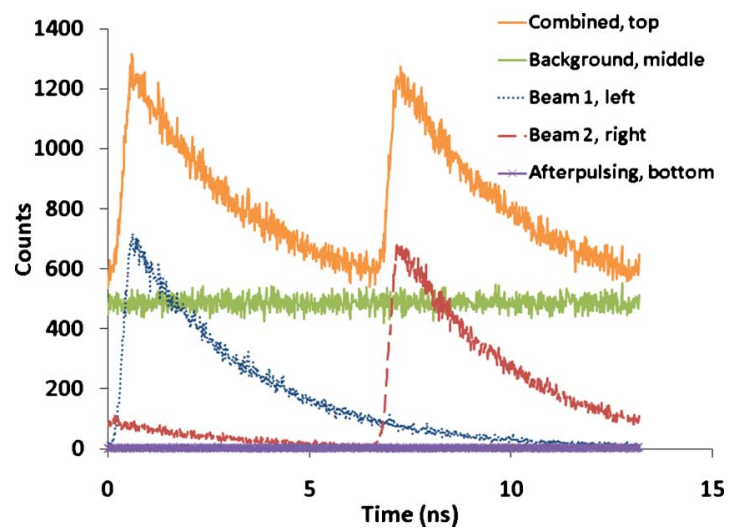

Fig. 2 Histograms of the timing delays (1024 channels at $12.89 \mathrm{ps} / \mathrm{channel}$ ) between the pulsed laser excitation (beam 1, left) and the detection of photons due to fluorescence from each beam (blue and red curves), background (green curve), detector afterpulses (purple curve), and all combined (orange curve), as collected during a simulation of sequential single-molecule trapping for a total simulated duration of $1000 \mathrm{~s}$. (Color online only.) 
During each move, if there are any molecules on the downstream end point of the grid, they will leave the simulation. Also, for each move, molecules may enter the simulation onto the upstream end point of the grid. To model this, note that molecules enter at random with a probability for entry for each grid step due to flow equal to $C_{0}$, the mean number of molecules per grid point. Thus, following the start of the simulation and whenever a molecule enters, a geometrically distributed random number for a probability of success $C_{0}$ is retrieved (using the Intel Math Kernel Library routine viRngGeometric) to find the number of flow steps until the next entry. The value of $C_{0}$ is given by

$$
C_{0}=10^{3} N_{A} C \Delta x d_{y} d_{z},
$$

where $N_{A}$ is Avogadro's number, $C$ is the molar concentration of molecules in solution, and $d_{y}, d_{z}$ are the width and depth of the nanochannel, with all lengths expressed in meters. Typical parameter values are $C=100 \mathrm{pM}$ and $d_{y}=d_{z}=10^{-7} \mathrm{~m}$, corresponding to $C_{0}=6 \times 10^{-6}$ molecules per grid point.

Brownian diffusion is independent of the electrokinetic flow and is modeled by Fick's second law of diffusion in one dimension:

$$
\frac{\partial \rho}{\partial t}=D \frac{\partial^{2} x}{\partial t^{2}} \rho
$$

where $\rho(x) \mathrm{d} x$ is the probability of finding a molecule within $\mathrm{d} x$ of $x$, and $D$ is the diffusion coefficient. With the initial condition of a molecule starting at the origin at time $t=0$ :

$$
\rho(x, t=0)=\delta(x),
$$

where $\delta(x)$ is the Dirac delta function, the solution of Eq. (7) is

$$
\rho(x, t) \mathrm{d} x=\frac{1}{\sqrt{2 \pi} \sigma(t)} \exp \left[\frac{-x^{2}}{2 \sigma^{2}(t)}\right] \mathrm{d} x,
$$

which is a normalized Gaussian distribution with standard deviation

$$
\sigma(t)=\sqrt{2 D t} .
$$

To model diffusion on a grid, molecules may hop to nearby grid points at regular time intervals $\Delta t_{D}$. The time step for diffusion is chosen so that the standard deviation of the Gaussian in Eq. (9) is one grid point, i.e., $\sigma\left(\Delta t_{D}\right)=\Delta x$, or

$$
\Delta t_{D}=(\Delta x)^{2} /(2 D),
$$

where $\rho\left(x, \Delta t_{D}\right) \mathrm{d} x=\exp \left(-x^{2} / 2\right) \mathrm{d} x / \sqrt{2 \pi}$. At each time step $\Delta t_{D}$, each molecule within the simulation is moved $j$ grid spaces, where $j$ is a random integer. To choose the value of $j$ with the appropriate Gaussian weighting, a 32-bit random number $X$ uniformly distributed between 0 and 1 is retrieved and successively compared with the cumulative probability values $P(j)$ listed in column 3 of Table 1 , beginning at the top, until it is found to be less than the value listed for the corresponding value of $j$. For example, if $X=0.6753$, then the value of $j$ is taken to be -1 because $0.6246<0.6753$
Table 1 Cumulative probabilities for diffusion.

\begin{tabular}{lll}
\hline$i$ & $P(j)$ expression & $P(j)$ value \\
\hline 0 & $2 E(0.5)$ & 0.382924922548026 \\
1 & $E(0.5)+E(1.5)$ & 0.624655260005155 \\
-1 & $2 E(1.5)$ & 0.866385597462284 \\
2 & $E(1.5)+E(2.5)$ & 0.926983133405366 \\
-2 & $2 E(2.5)$ & 0.987580669348448 \\
3 & $E(2.5)+E(3.5)$ & 0.993557705595188 \\
-3 & $2 E(3.5)$ & 0.999534741841929 \\
4 & $E(3.5)+(4.5)$ & 0.999763973247840 \\
-4 & $2 E(4.5)$ & 0.999993204653751 \\
5 & $E(4.5)+E(5.5)$ & 0.999996583337313 \\
-5 & $2 E(5.5)$ & 0.999999962020875 \\
6 & $E(5.5)+E(6.5)$ & 0.999999980970278 \\
-6 & $2 E(6.5)$ & 0.999999999919680 \\
\hline
\end{tabular}

$<0.8664$. The values in column 3 are given by the expressions in column 2 , where we define

$$
E(a)=\int_{0}^{a} \exp \left(-x^{2} / 2\right) \mathrm{d} x / \sqrt{2 \pi}
$$

and the expressions are evaluated using the error function:

$$
\operatorname{erf}(x)=2 E(\sqrt{2} x)
$$

Note that the probability to diffuse by more than six grid spaces is less than one part in $2^{32}$, and hence for the 32-bit random numbers used here, $j$ is between -6 and 6 .

When molecules hop to new grid points, there is a chance that some may hop off the grid and leave the simulation, but there is an equal possibility that new molecules may diffuse onto the grid. This is accounted for as follows: For each diffusion time step $\Delta t_{D}$, the probability that a new molecule hops onto a point $k$ spaces from the end of the grid is

$$
P_{k}=C_{0}[E(6.5)-E(0.5+k)], \quad k=0, \ldots, 5 .
$$

Note that this exactly balances the probability to leave the grid. For example, if a molecule is at a point $k=4$ spaces from the end of the grid, it can escape from the grid by hopping 5 or 6 spaces, with a probability $[E(5.5)-E(4.5)]+[E(6.5)$ $-E(5.5)]$. The total probability per diffusion time step $P$ that a new molecule enters somewhere onto the grid from either end is found by adding the probabilities to hop to a point that is $k=0$ to 5 spaces from either of the ends. This is hence given by 


$$
\begin{aligned}
P & =2 \sum_{k=0}^{5} P_{k}=2 C_{0}\left[6 E(6.5)-\sum_{k=0}^{5} E(0.5+k)\right] \\
& =0.763540130047191 C_{0} .
\end{aligned}
$$

Because molecules enter onto the grid at random at a rate equal to $P / \Delta t_{D}$, at the start of the simulation and whenever a new molecule enters by diffusion, a geometrically distributed random number for a probability of a success $P$ is retrieved to determine the number of diffusion time steps until the next entry. Also, a 32-bit uniform random number $X$ is retrieved and compared successively to the cumulative probabilities $Q_{l}$ listed in Table 2 to determine the position $l$ on the grid at which the molecule will enter.

Note that molecules may enter or leave the simulation from either end of the grid, but when the trap is operating, they are more likely to enter from the left $(l=-L=-1000$, or $x=-1000 \Delta x)$ and leave from the right, because when there is no fluorescence signal from molecules within the focused laser beams, the electrokinetic flow is set to a maximum so as to transport molecules in from the left side and out from the right side.

Experiments in our lab ${ }^{13}$ have found that diffusion of fluorescent dye molecules in a nanochannel fabricated from fused silica is slowed by a factor of $\sim 50$, in agreement with the observations of Lyon and Nie. ${ }^{15}$ However, it is likely that this is due to sticking of molecules to the nanochannel walls and that avoidance of sticking by surface treatment or other means will restore the free solution diffusion. Hence, to assess the capability for trapping molecules that do not stick, for the simulation results presented in Sec. 3, the diffusion coefficient is taken to be $D=2.2 \times 10^{-6} \mathrm{~cm}^{2} \mathrm{~s}^{-1}$ (with corresponding $\left.\Delta t_{D}=0.45 \mu \mathrm{s}\right)$, which is that of a small fluorescent dye mol-

Table 2 Cumulative probabilities for new entry positions.

\begin{tabular}{lll}
\hline$I$ & $Q_{1}$ expression & $Q_{1}$ value \\
\hline$-L$ & $Q_{0}=P_{0} / P$ & 0.404066599711270 \\
$L$ & $Q_{L}=Q_{0}+P_{0} / P$ & 0.808133199422541 \\
$1-L$ & $Q_{1}=Q_{L}+P_{1} / P$ & 0.895625174513805 \\
$L-1$ & $Q_{L-1}=Q_{1}+P_{1} / P$ & 0.983117149605069 \\
$2-L$ & $Q_{2}=Q_{L-1}+P_{2} / P$ & 0.991249444832068 \\
$L-2$ & $Q_{L-2}=Q_{2}+P_{2} / P$ & 0.999381740059067 \\
$3-L$ & $Q_{3}=Q_{L-2}+P_{3} / P$ & 0.999686395503419 \\
$L-3$ & $Q_{L-3}=Q_{3}+P_{3} / P$ & 0.999991050947771 \\
$4-L$ & $Q_{4}=Q_{L-3}+P_{4} / P$ & 0.999995500604795 \\
$L-4$ & $Q_{L-4}=Q_{4}+P_{4} / P$ & 0.999999950261819 \\
$5-L$ & $Q_{5}=Q_{L-4}+P_{5} / P$ & 0.999999975130910 \\
$L-5$ & $Q_{L-5}=Q_{5}+P_{5} / P$ & 1.000000000000000 \\
\hline
\end{tabular}

Note: $2 L+1=2001$ is the number of grid points. ecule with a hydrodynamic radius of $\sim 1 \mathrm{~nm}$ freely diffusing in solution. ${ }^{24}$ As expected, trapping is easier to achieve for smaller values of $D$.

\subsection{Photophysics}

As shown in Fig. 3, when irradiated, a molecule can become excited from the ground state $S_{0}$ to the $S_{1}$ manifold, and from here, it may follow four possible paths. It may (i) decay back to the ground state without the detection of a fluorescence photon, with probability $P_{(i)}$ (due either to nonradiative decay or fluorescence emission of a photon that is not detected); or (ii) decay back to the ground state with the detection of a fluorescence photon, with probability $P_{(i i)}$; or (iii) cross to the triplet manifold $T_{1}$ before decaying back to the ground state, with probability $P_{(i i i)}$; or (iv) undergo irreversible photobleaching and be removed from subsequent photophysical transitions, with probability $P_{(i v)}$. For (i) and (ii), relaxation back to the ground state occurs after a random time with an exponential distribution and mean equal to the fluorescence lifetime $\left(\tau_{f} \sim 3.0 \mathrm{~ns}\right)$, whereas for (iii), the mean is equal to the phosphorescence lifetime $\left(\tau_{p} \sim 1 \mu \mathrm{s}\right)$. The timing of excitation events, the pathway taken after excitation, and the time taken for relaxation to the ground state and possible photon detection are all stochastic processes that are modeled using Monte Carlo methods.

For trapping, the position determination of the molecule is dependent on the timing of photon detection events, which in turn is dependent on the timing of molecular excitation events. Two different methods have been used in the simulation to model the timing of molecular excitations. In the first, for each laser pulse (i.e., for each time step of $T / 2=6.6 \mathrm{~ns}$ ), a uniform random number is retrieved and compared to the probability for excitation per laser pulse for that beam to determine whether that pulse causes excitation. In the second, the waiting time until the next excitation is found as follows: Geometrically distributed random numbers for probabilities of success equal to the probabilities of excitation per laser pulse for each of the two beams are retrieved to find the times at which each beam would next give excitation, and the earlier event is then chosen. However, if the molecule moves due to diffusion or flow before excitation occurs, then new geometrically distributed random numbers are retrieved for the excitation probabilities per pulse appropriate for the new location of the molecule. We find that the second method generates results that are the same as the first but is computationally much

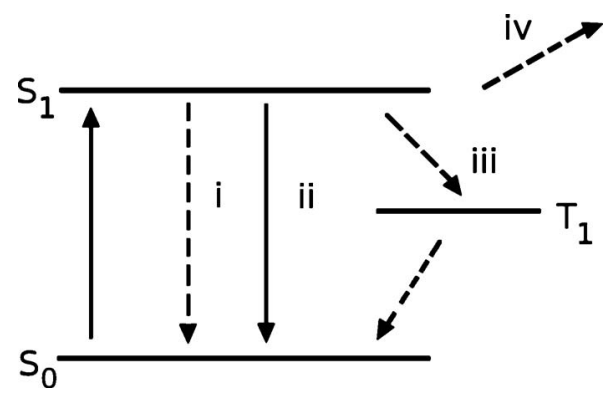

Fig. 3 Jablonski diagram for the decay possibilities of the molecule. $S_{0}$ is the singlet ground state, $S_{1}$ is the singlet excited state, and $T_{1}$ is the triplet state. 
faster, as may be expected because the mean time between excitation events is long compared to the interval between laser pulses $T / 2=6.6 \mathrm{~ns}$, yet short compared to $\Delta t_{F}$ and $\Delta t_{D}$.

For either method of simulating excitation, a molecule at position $x$ has a rate of excitation from each of the two beams $(b=1,2)$ given by

$$
k_{b}(x, t)=\sigma_{a} I_{b}(x, t) / E_{\lambda},
$$

where $\sigma_{a}$ is the absorption cross section, $E_{\lambda}$ is the photon energy, and $I_{b}(x, t)$ is given by Eq. (2) and (3). The probability for excitation for each laser pulse is thus $P_{1}^{E}(x)$ $=k_{1}(x, 0) T$ and $P_{2}^{E}(x)=k_{2}(x, T / 2) T$ for beams 1 and 2 . The values of these probabilities for each grid point may be stored in lookup tables. For the first method, these are then used for comparison with uniform random numbers to determine whether excitation occurs for each laser pulse. For the second method, these are then used to retrieve geometrically distributed random numbers for these probabilities of success to determine when the next excitation would occur. For the results presented in Sec. 3, parameter values are $\sigma_{a}=2$ $\times 10^{-16} \mathrm{~cm}^{2}$ (corresponding to a fluorophore such as Alexa $610), \quad E_{\lambda}=3.3 \times 10^{-19} \mathrm{~J}$, and a total laser power of $\bar{P}$ $=30 \mu \mathrm{W}$, so that the total mean excitation rate for a trapped molecule is $k_{1}(0,0)+k_{2}(0, T / 2) \approx 2.8 \times 10^{6} \mathrm{~s}^{-1}$.

Once a molecule is excited, a uniform random number $X$ is retrieved and successively compared to the summed values of the probabilities for the four possible relaxation pathways, which are listed in Table 3. If $X<P_{(i)}$, relaxation is by pathway (i); otherwise, if $X<P_{(i)}+P_{(i i)}$, relaxation is by pathway (ii); otherwise, if $X<P_{(i)}+P_{(i i)}+P_{(i i i)}$, relaxation is by pathway (iii); otherwise, relaxation is by pathway (iv).

The most likely pathway is (i) decay to the ground state without detection of a fluorescence photon, which occurs with a probability of $P_{(i)}=1-P_{(i i)}-P_{(i i i)}-P_{(i v)}$ (typically, $\sim 0.95$ ). This is the sum of the probability for nonradiative decay from $S_{1}$ and radiative decay with missed photon detection. The second most likely pathway is (ii) decay to the ground state with detection of a fluorescence photon. For a well-designed single-molecule microscope and for the results presented in Sec. 3, $P_{(i i)}=0.05$, and in practice, it is determined by the product of the fluorophore quantum efficiency $(\sim 0.8)$, the fluorescence collection efficiency of the microscope objective $(\sim 0.20)$; the transmission of the spectral filter, objective lens, and other optical components $(\sim 0.5)$; and the photon detec-

Table 3 Relaxation pathway probabilities.

\begin{tabular}{lll}
\hline Path & Mechanism & Probability \\
\hline i & $\begin{array}{l}\text { Singlet decay } \\
\text { without photon } \\
\text { detection }\end{array}$ & $\begin{array}{l}P_{(i)}=1-P_{(i i)} \\
-P_{(i i i)}-P_{(i v)}\end{array}$ \\
ii & $\begin{array}{l}\text { Singlet decay with } \\
\text { photon detection }\end{array}$ & $P_{(i i)}=0.05$ \\
iii & Triplet crossing & $P_{(i i i)}=10^{-3}$ \\
iv & Photobleaching & $P_{(\text {iv) }}=10^{-5}$ \\
\hline
\end{tabular}

tion efficiency of the SPAD detector $(\sim 0.65)$. The third most likely pathway is (iii) crossing to the triplet manifold, which is taken to be $P_{(i i i)}=10^{-3}$, and the least likely pathway is (iv) photobleaching, which is taken to be $P_{(i v)}=10^{-5}$. These last two values are typical parameters for fluorophores commonly used in single-molecule experiments. ${ }^{25}$ For the preceding probabilities and a mean excitation rate of $2.8 \times 10^{6} \mathrm{~s}^{-1}$, the expected photon count rate is $1.4 \times 10^{5} \mathrm{~s}^{-1}$, and the mean time before photobleaching is $\sim 35 \mathrm{~ms}$.

\subsection{Photon Detection}

The desired outcome of molecular excitation is photon detection, which occurs in relaxation pathway (ii). Whenever pathway (i) or (ii) is chosen, a random number with exponential distribution with mean equal to the fluorescence lifetime $\tau_{F}$ is retrieved to find the time of decay of the molecule, as discussed in the first paragraph of Sec. 2.3. For pathway (ii), the detection time of the photon is then found by adding another random number with a Gaussian distribution (with standard deviation of $\sigma=127.4$ ps and a mean of $3 \sigma$, in order to model the timing jitter of the SPAD and the setting of the time gate of the detection electronics of the apparatus of Ref. 13), as discussed at the end of Sec. 2.1 and in Fig. 2.

In addition to fluorescence photons, there are background photons due to detector dark noise $\left(d=50\right.$ photons $\left.\mathrm{s}^{-1}\right)$ and scattered light that passes through the filters ( $s$ $=50$ photons $\left.\mu \mathrm{W}^{-1} \mathrm{~s}^{-1}\right)$. In the results presented in Sec. 3, the total background count rate is taken to be $B=s \bar{P}+d$, which gives 500 photons s ${ }^{-1}$ for $\bar{P}=30 \mu \mathrm{W}$, and background is assumed to be random with Poissonian statistics. Hence, to simulate background, the stochastic time of occurrence of the next background photon is determined by retrieving an exponentially distributed random number with a mean equal to the reciprocal of the background count rate.

Whenever the simulation finds the time of occurrence for (1) the next background photon, (2) the next flow time step, (3) the next resetting of the flow direction during trapping, (4) the next diffusion time step, or (5) the next photophysics event for any of the molecules in the simulation (possible excitation, decay without photon detection, or decay with photon detection), it then finds the process with the minimum time (using the Intel Math Kernel Library routine idamin). The simulation proceeds with whatever process occurs first and then generates the time for the next occurrence of that process. In this way, independent processes are synchronized.

Whenever a photon is detected (either background or fluorescence), its time of arrival is determined by recording the total number of laser pulses (with period $T / 2$ ) since the beginning of the experiment. This time-stamp $t s_{i}$, or laser-pulse count at the time of detection of the $i$ 'th photon, which is stored as a 32-bit unsigned integer for compatibility with the analysis routines for the experiments of Ref. 13, is either even or odd, depending on whether the photon detection follows a laser pulse to the left or right of the center of the trap. Thus, time-gated photon detection may be achieved by sorting photons based on the value of the least significant bit of the timestamp.

Note also that the SPAD detector has a dead time of $\sim 40 \mathrm{~ns}$, during which it is unresponsive. This is modeled by 
ignoring a photon if it follows another by less than the SPAD dead time. Also, for each photon detected, there is a $0.5 \%$ probability that the SPAD detector will experience an afterpulse, in which case, another photon detection event will occur a random time later with an exponential distribution with a mean of $\sim 100 \mathrm{~ns}$ (Ref. 26). These processes are also included in the simulation and affect the shape of the autocorrelation function for small delays, but they have been found to not significantly affect the performance of the trapping for the parameters of Sec. 3 .

\subsection{Trapping}

Each time a photon is detected, a routine is entered for adjustment of the electrokinetic flow in order to achieve rapid delivery and trapping of individual molecules. A significant purpose of the simulation is to evaluate the effectiveness of the algorithm used by this routine when subject to restrictions imposed by the experimental hardware. The main constraint is that the maximum electrokinetic flow speed is limited, and for most of the results of Sec. 3, it is taken to be $v_{F}^{\max }$ $=2 \mu \mathrm{m} / \mathrm{ms}$, which corresponds to $\Delta t_{F}=5 \mu \mathrm{s}$. In the apparatus of Ref. 13, it is possible to apply a potential of $10 \mathrm{~V}$ across a 200- $\mu \mathrm{m}$-long nanochannel to achieve a field of 5 $\times 10^{4} \mathrm{~V} / \mathrm{m}$, whereas electrokinetic speeds of single molecules in capillaries are estimated to be in the range of 2 to $6 \mu \mathrm{m} / \mathrm{ms}$ for similar fields. ${ }^{27}$ In the $2-\mathrm{D}$, ABEL trap, a maximum electrokinetic speed of $3 \mu \mathrm{m} / \mathrm{ms}$ has been reported. Also, another potential constraint is that there is a delay or latency for adjustment of the flow due to the response time of the FPGA electronics and the switching time of the voltage applied to the nanochannel. For the apparatus of Ref. 13 , the latency is $\Delta t_{L}=6 \mu \mathrm{s}$, which is comparable to $\Delta t_{F}$, and as expected, this has been found to not significantly affect trapping. Nevertheless, to study the effects of latency, whenever the algorithm makes an adjustment to the flow velocity (and hence to the flow time step $\Delta t_{F}$ ), the change is scheduled to occur with a time delay of $\Delta t_{L}$. As presented in Sec. 3 , the performance of the trap degrades significantly if the latency is increased beyond $\sim 100 \mu \mathrm{s}$. The algorithm makes adjustments to the flow velocity, and hence the time of the next flow step based on the values of the time-stamps $t s_{i}$ of the last $N$ detected photons. For the results of Sec. 3, we have taken $N$ $=6$, in accord with the experiments of Ref. 13. Figure 4 shows a flowchart of the algorithm, which is explained in the following. A similar algorithm is programmed into the FPGA used in the experiments of Ref. 13, but in this case, the latency is not added in the algorithm, as it is already present in the electronics.

Before a molecule is transported into the laser foci so as to emit fluorescence photons, only background photons are detected. At this time, the electrokinetic flow is set to the maximum value $v_{F}^{\max }$ until the observed fluorescence signal is found to be above the background level. To make this determination with fast response, the difference between the last photon time-stamp and the one just two photons earlier must be shorter than a preset threshold time th, i.e.,

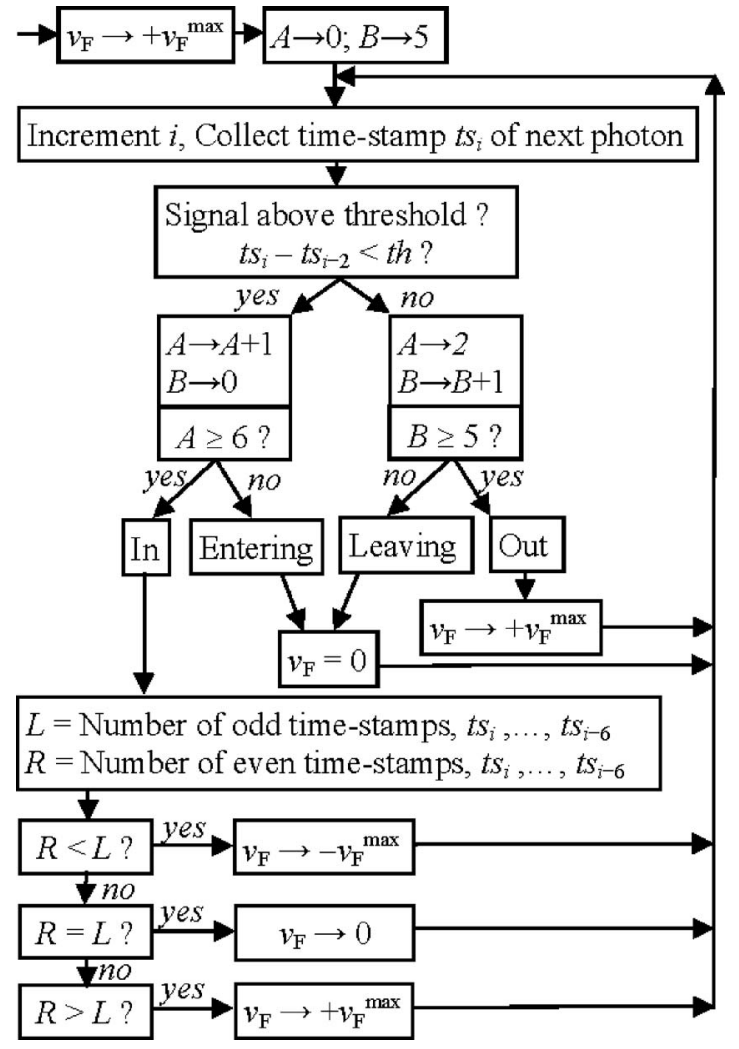

Fig. 4 Flowchart of the trapping algorithm.

$$
t s_{i}-t s_{i-2}<t h,
$$

where the threshold time is taken to be $t h=1 /(5 B T)$ $=30,303$, corresponding to a count rate of 2.5 times the background level. Although in principle it would be possible to use a longer threshold time, it is found that small variations of the threshold produce no perceptible difference in the performance of the trapping. Also, in experiments, the background may not be known accurately or may vary, and hence a lower threshold time is more suitable.

Once the photon signal is above the background level, it is assumed that a molecule has been transported into the detection zone, so after the latency delay $\Delta t_{L}$, the flow is adjusted to zero. Then, after $N-3$ more photons are detected and after the latency delay $\Delta t_{L}$, the flow is adjusted to a value dependent on the numbers of the last $N$ photons that are from each of the two laser beams. As before, each photon time-stamp is odd or even depending on whether the photon is more likely to originate from fluorescence excitation from the left or right laser focus. If more of the last $N$ photons have an odd (even) time-stamp, the molecule is assumed to be to the left (right) of the center of the trap, and the algorithm then schedules the flow to be to the right (left) with the maximum flow speed $v_{F}^{\max }$. If the numbers of odd and even time-stamps are equal, the algorithm then schedules the flow to be zero. The flow velocity is maintained until after the next scheduled change, which occurs $\Delta t_{L}$ after the next detected photon. For a fluorescence count rate of $\sim 10^{5}$ photons/s, the mean time between photons is $\sim 10 \mu \mathrm{s}$, and in this time, the molecule will be moved by flow only $\sim 0.02 \mu \mathrm{m}$ and so should remain 
within the trapping region, which is $\sim 0.5 \mu \mathrm{m}\left(2 x_{1}\right)$ in length. Also, during initial entry of a molecule into the trap, the flow is set to zero until a time $\Delta t_{L}$ after $N-3$ more photons are accumulated. The mean time for detection of $N-3$ photons is $\sim 10(N-3) \mu \mathrm{s}$, and in this time, the root-mean-square distance that a molecule diffuses is $\sim 0.2(N-3)^{1 / 2} \mu \mathrm{m}$, or $\sim 0.36 \mu \mathrm{m}$, so it is unlikely that the molecule diffuses through the trapping region in this time. Also, triplet blinking does not significantly increase the chances of escape from the trap, as the triplet decay time $(\sim 1 \mu \mathrm{s})$ is shorter than the mean time between photons $(\sim 10 \mu \mathrm{s})$. If escape from the trap occurs due to statistical fluctuations, it is most likely due to a series of incorrect estimates for the direction of applied electrokinetic flow.

If the signal level decreases to the level of the background so that the condition in Eq. (17) is not true, then it is likely that that the molecule has bleached or has escaped from the trap, but it is also possible that this may occur due to statistical fluctuations. To avoid ejecting a molecule from the trap because of such fluctuations, the flow velocity is set to zero, and the condition in Eq. (17) must be false $S=5$ times in succession before the algorithm steps out of the trapping loop and sets $v_{F}=v_{F}^{\max }$ until the next molecule is loaded into the trap. The inclusion of this period of time with flow velocity set to zero following loss of signal and of multiple testing before reloading is found to reduce the probability for escape and to provide an opportunity for recapture if there is escape (as discussed with Fig. 5).

\section{Results and Discussion}

Table 4 summarizes the typical values of the parameters used in the simulations presented in this section. A simulation of a 1000 -s experiment, including collation of statistical and diagnostic information, executes within $\sim 120 \mathrm{~s}$ on a $2.3-\mathrm{GHz}$ Dual-Core Pentium PC. Thus, repeated execution of the pro-

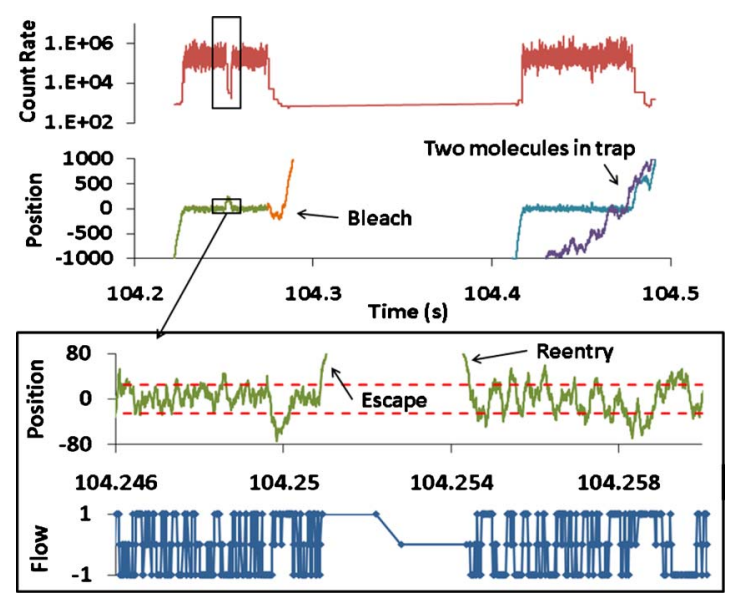

Fig. 5 Example of the total photon count rate $R(t)$ (first plot, red), and molecule trajectories $x(t)$ (second plot); (position given in units of grid spaces, $\Delta x=0.01 \mu \mathrm{m}$ ) during a simulation of trapping using parameters in Table 4. A small section of the trajectory data is expanded in the inset. The red dashed lines indicate the centers of the laser foci $(x= \pm 0.25 \mu \mathrm{m})$. The lower plot in the inset shows the changes to the flow direction imposed by the trapping algorithm during the same time. (Color online only.)
Table 4 Simulation parameters.

\begin{tabular}{|c|c|}
\hline Parameter & Value \\
\hline Laser power $\bar{P}$ & $30 \mu \mathrm{W}$ \\
\hline Beam waist $\omega_{0}$ & $0.5 \mu \mathrm{m}$ \\
\hline Laser foci separation $2 x_{1}$ & $0.5 \mu \mathrm{m}$ \\
\hline Laser wavelength $\lambda$ & $610 \mathrm{~nm}$ \\
\hline Laser pulse spacing $T / 2$ & $6.6 \mathrm{~ns}$ \\
\hline Absorption cross section $\sigma_{a}$ & $2 \times 10^{-16} \mathrm{~cm}^{2}$ \\
\hline Fluorescence lifetime $\tau_{f}$ & $3.0 \mathrm{~ns}$ \\
\hline Triplet lifetime $\tau_{p}$ & $1.0 \mu \mathrm{s}$ \\
\hline Grid resolution $\Delta x$ & $0.01 \mu \mathrm{m}$ \\
\hline Grid length $2 L \Delta x$ & $20 \mu \mathrm{m}$ \\
\hline Diffusion coefficient $D$ & $220 \mu \mathrm{m}^{2} / \mathrm{s}$ \\
\hline Maximum speed $\nu_{F}^{\max }$ & $2.0 \mu \mathrm{m} / \mathrm{ms}$ \\
\hline Background count rate $B$ & $500 / s$ \\
\hline Concentration $C$ & $100 \mathrm{pM}$ \\
\hline Feedback latency $\Delta t_{L}$ & $6 \mu \mathrm{s}$ \\
\hline
\end{tabular}

gram allows one to optimize experimentally adjustable parameters such as the laser power, to easily modify experimentally fixed parameters to study limitations imposed by processes such as detector dead time, background, photobleaching, triplet kinetics, and control latency, and also to view information that would not be readily available in an experiment, such as the trajectory and photophysical state of each molecule.

Figure 5 presents an example of the photon count rate $R(t)$ and the positions $x(t)$ of molecules during operation of the trapping algorithm with parameters from Table 4, during a 0.3 -s interval from time $t=104.2 \mathrm{~s}$ to $104.5 \mathrm{~s}$. The count rate shown in the figure is updated with each detected photon time-stamp but is averaged over only the last $N=6$ detected photons: $R\left(t s_{i}\right)=N /\left[(T / 2)\left(t s_{i}-t s_{i-N}\right)\right]$. Hence, it exhibits considerable statistical fluctuations and so is plotted on a semilogarithmic scale. During the selected 0.3-s interval, individual molecules are brought into the detection volume by the electrokinetic flow and are trapped, but in this particular slice of data, several unusual occurrences are also seen. After the first molecule (in Fig. 5) is carried in by flow, the count rate increases to $>10^{5} \mathrm{~s}^{-1}$, and the molecule is trapped. Then, the count rate suddenly dips and the signal falls below threshold, i.e., Eq. (17) fails, as the molecule escapes the trap (data shown within black rectangles in Fig. 5).

The inset in Fig. 5 shows an expansion of the trajectory data around this time of escape. The red dashed lines are the positions of the centers of the two laser foci, between which the molecule is to be trapped. The direction of the electroki- 
netic flow, which is determined by the trapping algorithm from the difference in the numbers of the last $N=6$ photons that have odd and even time-stamps, is also shown in the inset. Note that at the time of escape, the flow is set in the incorrect direction. After the escape, the flow is set to zero and the molecule fortunately diffuses back into the excitation region and is trapped once again, as the algorithm reactivates switching of the flow direction in response to the difference in odd and even photon time-stamps. By comparing the two plots in the inset, one can see how the flow is adjusted to recenter the molecule, albeit with considerable error due to the shot noise from the low number of photons used for analysis. For example, near the end of the data in the inset, the molecule position is $x \approx+40 \Delta x$ and the flow velocity is mostly $v_{F}=-v_{F}^{\max }$, which is in the correct direction to bring the molecule back to the center. However, during simulations of experiments of 1000-s duration, due to shot noise, the fraction of the time that the electrokinetic velocity is in the incorrect direction is found to be $\sim 0.25$. Also, molecules often pass beyond the region between the centers of the two laser foci, and the fraction of molecules that permanently escape the trap and leave the simulation before photobleaching is found to be $\sim 0.17$.

In the second plot of Fig. 5, it is shown that the first molecule photobleaches at the point where the green line changes to orange. Soon after this point, the flow is switched to zero and the photobleached molecule diffuses freely for a brief time, but the count rate does not recover and hence the algorithm then switches the flow velocity to $v_{F}=+v_{F}^{\max }$, so the photobleached molecule is transported in the positive direction out of the simulation. Approximately $0.1 \mathrm{~s}$ later, a second molecule, shown by a light blue trajectory, is transported by the flow into the detection volume and is subsequently trapped. While the flow direction is alternating to hold this molecule trapped, a third molecule, shown by a purple trajectory, diffuses into the simulation volume. By chance, at $t$ $\approx 104.47 \mathrm{~s}$, this diffuses into the laser foci, and the count rate approximately doubles (although this is difficult to discern on the logarithmic scale). The algorithm responds to the counts from both molecules but cannot keep two independently diffusing molecules at the center of the trap. By chance, the third molecule diffuses away, and the second molecule remains trapped for a short while later, and then it too escapes from the trap and diffuses away.

The study of simulated trajectory data such as that in Fig. 5 can provide insight on the effectiveness and/or the causes of failings of the trapping algorithm and thereby lead to more complicated algorithms with improved performance. Experiments cannot provide such detailed data, but they do provide statistical information about the trap performance from the normalized autocorrelation function $g(\tau)$ of the stream of detected photons. ${ }^{13}$ The amplitude and width of this function provide information about the mean number and residency time of molecules within the detection volume. The amplitudes, widths, shapes, and trends of the autocorrelation functions from the experimental runs presented in Ref. 13 are consistent with those generated by simulations, as presented in the following.

Figure 6 presents $g(\tau)$ for simulated experiments of 1000 -s duration with parameters given in Table 4 but with

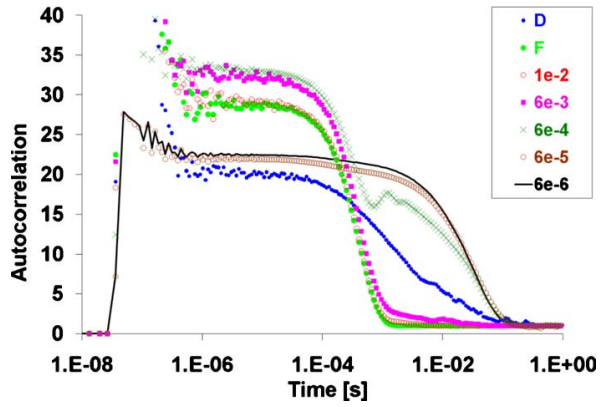

Fig. 6 Autocorrelation functions for a laser power of $\bar{P}=30 \mu \mathrm{W}$ for free diffusion ( $\mathrm{D}$, blue) and for constant electrokinetic flow ( $\mathrm{F}$, green) and for trapping for a range of values for the feedback latency from $1 \times 10^{-2} \mathrm{~s}$ to $6 \times 10^{-6} \mathrm{~s}$. (Color online only.)

different latency delays of the feedback for the trap, decreasing down to $\Delta t_{L}=6 \times 10^{-6} \mathrm{~s}$, which is that of the electronics used in Ref. 13. Also shown are the plots of $g(\tau)$ for the cases of no trapping with (1) free diffusion, and (2) a constant electrokinetic flow at $v_{F}=+v_{F}^{\max }$. The change in shape and decrease in the width of $g(\tau)$ between these two curves are consistent with the experimental results shown in Figs. 14 and 15 of Ref. 13. For all these plots, $g(\tau)$ is obtained directly from the sequence of time-stamps of detected photons by use of a separate software correlator program written in LabView, ${ }^{22}$ which is also used in the analysis of experimental data. For a latency delay of $\Delta t_{L}=1 \times 10^{-2} \mathrm{~s}$, the autocorrelation function is almost identical to that of the constant flow case, meaning that the trapping behavior is completely broken. On the other hand, for a latency of $\Delta t_{L}=6 \times 10^{-6} \mathrm{~s}$, the width of the autocorrelation is extended beyond that of free diffusion, out to a width of about $35 \mathrm{~ms}$, the mean time before photobleaching calculated at the end of Sec. 2.3, indicating that the trap is working. For a latency of $\Delta t_{L}=6 \times 10^{-5} \mathrm{~s}$, the autocorrelation is almost the same, but when the latency is increased to $\Delta t_{L}=6 \times 10^{-4} \mathrm{~s}$, the trap begins to fail, as the autocorrelation now contains both the trapped and constant flow components. There is also a fluctuation at a delay time of $\sim 6 \times 10^{-4} \mathrm{~s}$, due to molecules being driven out and then back into the detection volume by the electrokinetic flow.

For all the plots in Fig. 6, there is structure for time delays less than $\sim 10^{-6} \mathrm{~s}$, also seen in experimental $g(\tau)$, due to detector dead time and afterpulses. If the values for these parameters are altered in simulations, the structure also changes. Beyond these features, at a time delay of $\sim 10^{-5} \mathrm{~s}$, the amplitude of the shoulder gives an indication of $\bar{N}$, the mean number of molecules within the detection volume during the course of the simulated experiment. In the fluorescence correlation spectroscopy (FCS) literature, the amplitude is usually taken to be inversely proportional to $\bar{N}$, although it is also proportional to $(1-B / S)^{2}$, where $S / B$ is the signalto-background ratio. ${ }^{22}$ One would normally expect the amplitudes for free diffusion and constant flow to be the same, as $\bar{N}$ is the same [as $C_{0}$ molecules per grid point given in Eq. (6) is the same]. However, for free diffusion, $\bar{N}$ has large fluctuations due to molecular shot noise, even for a run time of $1000 \mathrm{~s}$. If the simulation is run with different random number 
seeds, the number of photon bursts from single molecules and the amplitude of $g(\tau)$ varies considerably from run to run $(13.2,17.8,23.9,18.3,20.1, \ldots)$. This is not the case for flow or trapping, as the number of molecules that pass through the volume is much larger, so the molecular shot noise becomes negligible. Also, for free diffusion, triplet crossing and photobleaching reduce the signal-to-background ratio, and this reduces the amplitude of the autocorrelation function. All of these features can be easily observed by running the simulation with different parameters, and they explain why the amplitude for flow is greater than that of the one plot shown for diffusion. In contrast, when the trap is operating, the reduction in the amplitude of $g(\tau)$ from that of constant flow is found to be because trapping effectively increases $\bar{N}$. The occupancy $\bar{N}$ increases, because soon after each molecule photobleaches or escapes from the detection volume, the flow is switched to quickly bring in the next molecule. A similar increase in width and reduction in amplitude of $g(\tau)$ when the trap is turned on is observed in experiments reported in Fig. 17 of Ref. 13. The simulations provide supporting evidence that the changes seen in the experiments are due to effective trapping and rapid replacement of molecules, which effectively increases the mean molecular occupancy within the confocal probe region.

Figure 7 presents $g(\tau)$ obtained from a study of the effects of laser power on the trapping. For reference, plots corresponding to no trapping with free diffusion and with a constant electrokinetic flow are also included. It is found that there is an optimum laser power of $\sim 30 \mu \mathrm{W}$ (plot shown in red), which produces the longest mean residence time [greatest width of $g(\tau)]$ and the largest molecular occupancy $\bar{N}$ [smallest amplitude of $g(\tau)$ ]. This is about the same value of laser power that was found to be optimal for the experiments presented in Ref. 13. If the laser power is adjusted between $\sim 20 \mu \mathrm{W}$ and $\sim 40 \mu \mathrm{W}$, the performance of the trap deteriorates only slightly from that at $30 \mu \mathrm{W}$. However, as the laser power is increased beyond $\sim 40 \mu \mathrm{W}$, photobleaching becomes more significant, so the mean residence time and the molecular occupancy are decreased [width of $g(\tau)$ decreases and amplitude increases]. Similarly, as the laser power is decreased below $\sim 20 \mu \mathrm{W}$, the rate of fluorescence photons falls, shot noise becomes more significant, more molecules

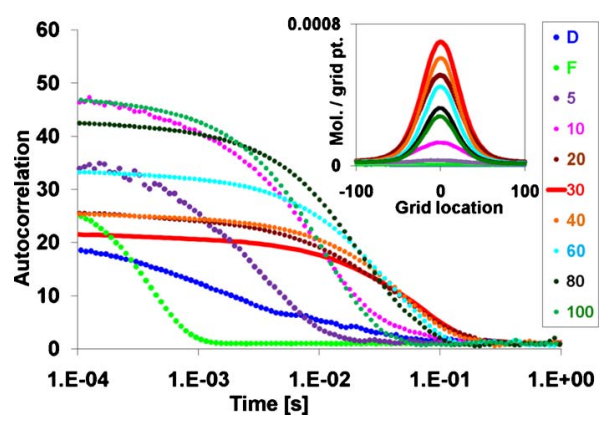

Fig. 7 Autocorrelation functions for free diffusion (D, blue) and for constant electrokinetic flow (F, green), each at a laser power of $\bar{P}$ $=30 \mu \mathrm{W}$, and for trapping for a range of laser powers from $5 \mu \mathrm{W}$ to $100 \mu \mathrm{W}$, all with feedback latency of $6 \times 10^{-6} \mathrm{~s}$. The inset shows the mean number of molecules per grid point $(\Delta x=0.01 \mu \mathrm{m})$ under the same conditions. (Color online only.) escape due to shot noise fluctuations, and the mean residence time and the molecular occupancy are decreased.

As the laser power is adjusted, in addition to the changes in the experimentally measureable autocorrelation, the inset of Fig. 7 shows that there are changes in the mean concentration of molecules in the detection volume. This would be difficult to directly measure experimentally but is easily obtained in the simulation by accumulating a histogram of molecular positions for each diffusion time step $\Delta t_{D}$. For constant flow, the concentration profile is constant at a value of $C_{0}=6 \times 10^{-6}$ molecules per grid point, whereas for diffusion, the profile exhibits molecular shot noise and varies spatially and from run to run around this value. When the trap is operating, the concentration profile has a peak at the origin, which is clearly visible in the graph. Note that the laser power of $30 \mu \mathrm{W}$ produces the tightest and tallest profile, with a peak of $\sim 7$ $\times 10^{-4}$ molecules per grid point, which is equivalent to a concentration increase by over a factor of 100 .

Some statistical data from the latency and laser power studies of Figs. 6 and 7 are shown in Fig. 8. In the simulation, one can follow each molecule individually to gather statistical information, such as the number of fluorescence photons that are detected from that molecule and the time between entry and exit from the detection volume. Figure 8 plots the mean occupancy time and the mean number of photons collected from each molecule against the laser power or the latency. This figure demonstrates again that a power of $30 \mu \mathrm{W}$ provides the longest occupancy time. Higher laser powers result in collection of a similar number of photons, but within a shorter occupancy time, due to faster photobleaching. The figure also demonstrates that for a laser power of $30 \mu \mathrm{W}$, the trapping is effective if the latency is below $\sim 1 \times 10^{-4} \mathrm{~s}$. In comparison, the mean time for acquisition of $N=6$ photons required by the algorithm is $\sim 4.3 \times 10^{-5} \mathrm{~s}$, and the mean time to diffuse out of the detection region is $\sim 6 \times 10^{-4} \mathrm{~s}$.

A study of the effects of power imbalance between the two laser beams was conducted using simulations. If there is a power imbalance of between about $+20 \%$ (i.e., $12 \mu \mathrm{W}$ and $18 \mu \mathrm{W}$ ) and $-5 \%$ (i.e., $15.75 \mu \mathrm{W}$ and $14.25 \mu \mathrm{W}$ ), the trap-

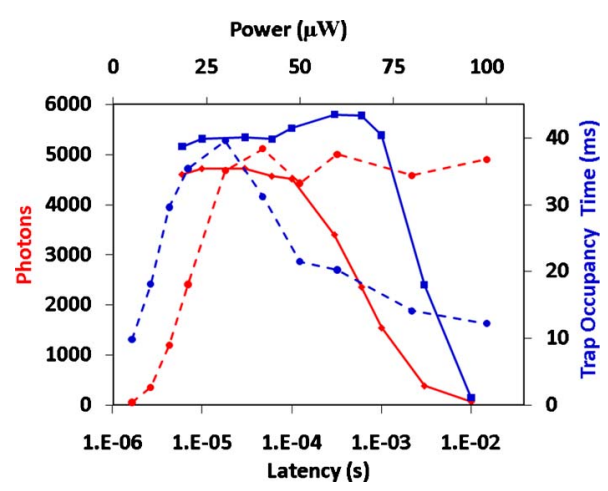

Fig. 8 Effect of laser power and latency of feedback on the trapping performance. The red curves show the mean number of photons detected (photons) before the molecule photobleaches or escapes versus latency (solid line, bottom scale) and versus power $\bar{P}$ (dashed line, top scale). The blue curves show the mean time that a molecule remains in the trap (trap occupancy time) versus latency (solid line) and power (dashed line). (Color online only.) 
ping is still effective in that the autocorrelation function and the statistics of the trapping time remain the same, but the center of the trap shifts slightly, as may be seen in the concentration profiles in Fig. 9. However, if the imbalance is higher, molecules escape from the low power end, and the trap is less effective. If a molecule escapes to the left, the trapping algorithm switches on the flow to reload the trap, and the molecule is brought back to the center and retrapped. Hence, an improvement in trapping statistics is found if the downstream laser beam has slightly higher power.

As discussed at the end of Sec. 2.1 (Fig. 2), good trapping depends on effective time-gated photon detection, and one expects temporal cross talk to increase and the trapping to become poor if the fluorescence lifetime of the molecule is long compared to the 6.6-ns interval between laser pulses. This is indeed the case, as seen in Fig. 10, which shows the mean number of photons and the mean trapping time per molecule as a function of fluorescence lifetime, and also the fraction of photons that have incorrect timing (odd time-stamp instead of even, or vice versa) as a function of fluorescence lifetime. The trapping performance deteriorates if the fluorescence lifetime is longer than about 3 ns. Also, simulations have verified the experimental finding that good trapping is dependent on correct timing delays (cable lengths) - i.e., the mean shift of $3 \sigma$ discussed at the end of Sec. 2.1 must be correctly set within the range of about $-0.5 \mathrm{~ns}$ to $+1.0 \mathrm{~ns}$, as seen in the inset of Fig. 10, which is for a fluorescence lifetime of $3 \mathrm{~ns}$.

Simulations indicate that the trapping is predicted to be effective for a wide range of sample concentrations from $<1 \mathrm{pM}$ up to $>0.1 \mathrm{nM}$, but as the concentration increases, trapped molecules are increasingly displaced by others that diffuse into the trap, as described with Fig. 5 (see top figure labeled "two molecules in trap"). This is especially the case for concentrations $>1 \mathrm{nM}$ or if photostability is improved. In an experiment that uses a concentration $>1 \mathrm{nM}$, it would be difficult to determine whether extended photon bursts are due to trapping of single molecules with enhanced photostability or to trapping of a series of molecules in succession. Also, as mentioned at the end of Sec. 2.2, simulations find that for a

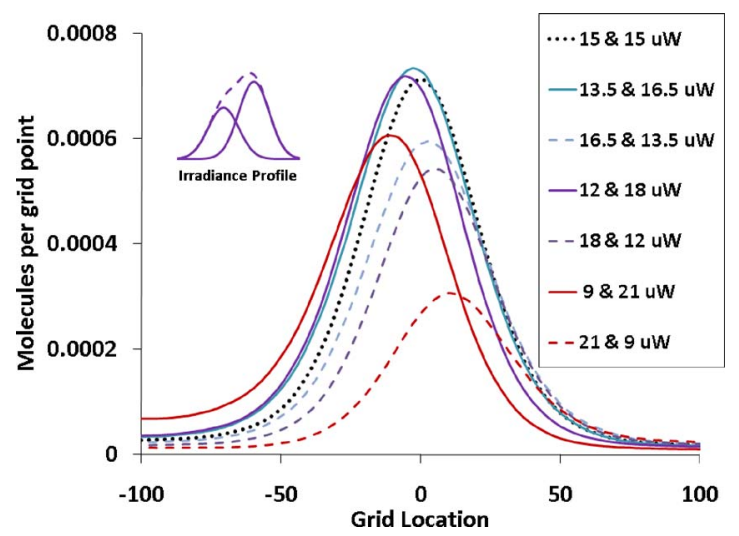

Fig. 9 Time-averaged concentration profile of trapped molecules or the mean number of molecules per grid point $(\Delta x=0.01 \mu \mathrm{m})$ for different values of laser powers $(\mu \mathrm{W})$ of the two beams. The inset shows the irradiance profile for a $+20 \%$ power imbalance, i.e., $12 \mu \mathrm{W}$ and $18 \mu \mathrm{W}$. smaller value of diffusion $D$, trapping is more easily achieved, i.e., parameters such as the fluorescence lifetime or timing delay may be varied over a wider range while still maintaining effective trapping.

The simulation results presented so far have assumed that an electrokinetic flow of $v_{F}^{\max }=2 \mu \mathrm{m} / \mathrm{ms}$ is achieved for the maximum applied voltage. This value was varied to study the predicted effectiveness of the trap for molecules with different electrokinetic mobilities. The autocorrelation width, which measures the mean residence time of molecules in the trap, remains about the same for values of $v_{F}^{\max }$ from 1 to $3 \mu \mathrm{m} / \mathrm{ms}$, while the amplitude decreases with $v_{F}^{\max }$, as might be expected for faster reloading of the trap, which increases the mean occupancy of the trap. However, for $v_{F}^{\max }$ $=0.5 \mu \mathrm{m} / \mathrm{ms}$ or lower, the autocorrelation width decreases as escape from the trap increases. The same applies also for $v_{F}^{\max }=4 \mu \mathrm{m} / \mathrm{ms}$ or higher, due to overcompensation of Brownian diffusion, and in this case, improved performance might be expected from a more sophisticated trapping algorithm that applies corrective voltages for limited time durations, so that molecules are not transported out of the trap in the time between photons.

\section{Conclusions}

The results show that single-molecule trapping in a nanochannel by control of electrokinetic flow to counteract Brownian diffusion is feasible for an experimental setup. A simple control algorithm that uses the timing of detected photons to determine adjustments of the flow for trapping is evaluated. While accommodating the limitations of a maximum electrokinetic flow of about $v_{F}^{\max }=2 \mu \mathrm{m} / \mathrm{ms}$ and a mean count rate of $\sim 1.4 \times 10^{5}$ photons $^{-1}$ from a molecule at the center of the detection volume, it is possible to hold a small rapidly diffusing molecule within a micron-sized confocal probe region for a prolonged time, usually until photobleaching occurs. The trap is robust in that it is possible to rapidly reload and trap a sequence of individual molecules over a broad

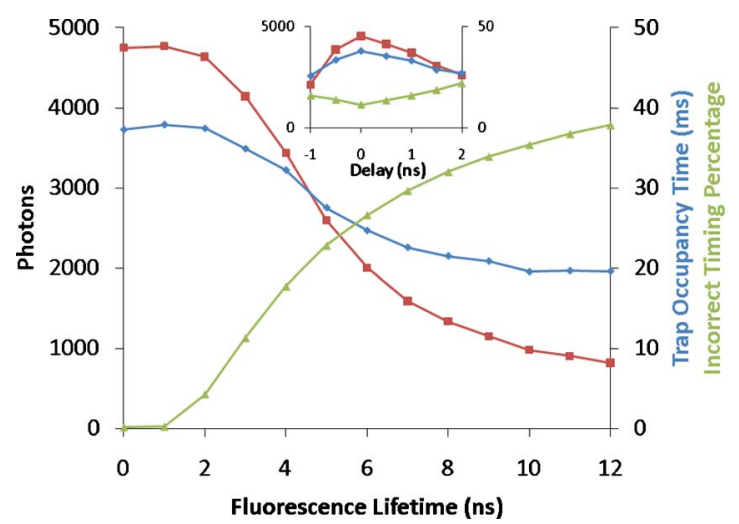

Fig. 10 Effect of fluorescence lifetime on the trapping performance. The red curve shows the mean number of photons detected (photons) before the molecule photobleaches or escapes, the blue curve shows the mean time that a molecule remains in the trap (trap occupancy time), and the green curve shows the percentage of fluorescence photons that have incorrect timing (incorrect timing percentage). The inset shows the effect of an incorrect timing delay on the trapping performance for a fluorescence lifetime of 3 ns. (Color online only.) 
range of experimentally achievable parameters. Studies with the simulation show that the anticipated latency of the control does not hinder performance and that trapping should be achievable provided the latency is below $\sim 100 \mu \mathrm{s}$.

By examination of the autocorrelation function of detected photons and collation of statistical data from individual molecules, it is found that there is a most favorable laser excitation power $(\sim 30 \mu \mathrm{W})$ for which the performance of the trap is optimum. If the laser power is reduced $\sim 50 \%$ below this point so that the fluorescence signal falls below $\sim 7$ $\times 10^{4}$ photons $\mathrm{s}^{-1}$, then the possibility of escape due to photon shot-noise fluctuations increases, and the trap begins to fail. Similarly, if the laser power is increased by $\sim 50 \%$ above this point, the rate of photobleaching increases so that the occupancy time of molecules within the trap is reduced, although the mean number of photons detected from each molecule is retained. The trap performance would improve if the rate of photobleaching were decreased, for example, by addition of oxygen scavengers to the solution. In preliminary experiments, molecules in nanochannels appear to have improved photostability compared to those in microchannels or in bulk solution. ${ }^{13}$

The algorithms for simulating single-molecule detection reported in this paper are applicable to a wide variety of experiments, including studies of molecular interactions for high-throughput screening. ${ }^{28}$ An extension of the simulation to study trapping with two-photon excitation and also trapping in three dimensions is under way. The approach involves use of four laser foci arranged in a tetrahedron to provide 3-D spatial information and also four electrodes arranged in a tetrahedron to provide $3-\mathrm{D}$ electrokinetic motion. ${ }^{17}$

\section{Acknowledgments}

We thank Zbigniew Sikorski for helpful discussions in the early part of this work, and acknowledge support from DARPA Grant No. W911NF-07-1-0046, NIH Grant No. EB006639, NSF Grant No. 0619789, and the Center for Laser Applications. The contents of this paper are solely the responsibility of the authors and do not necessarily represent the official views of the NIH.

\section{References}

1. J. Enderlein, "Tracking of fluorescent molecules diffusing within membranes," Appl. Phys. B 71, 773-777 (2000).

2. A. J. Berglund and H. Mabuchi, "Tracking-FCS: fluorescence correlation spectroscopy of individual particles," Opt. Express 13, 80698082 (2005).

3. K. Kis-Petikova and E. Gratton, "Distance measurement by circular scanning of the excitation beam in the two-photon microscope," Microsc. Res. Tech. 63, 34-49 (2003).

4. V. Levi and E. Gratton, "Exploring dynamics in living cells by tracking single particles," Cell Biochem. Biophys. 48, 1-15 (2007).

5. G. A. Lessard, P. M. Goodwin, and J. H. Werner, "Three-dimensional tracking of individual quantum dots," Appl. Phys. Lett. 91, 224106 (2007).

6. N. P. Wells, G. A. Lessard, and J. H. Werner, "Confocal, threedimensional tracking of individual quantum dots in high-background environments," Anal. Chem. 80, 9830-9834 (2008).
7. C. Gosse and V. Croquette, "Magnetic tweezers: micromanipulation and force measurement at the molecular level," Biophys. J. 82, 33143329 (2002).

8. A. Ashkin, "Optical trapping and manipulation of neutral particles using lasers," Proc. Natl. Acad. Sci. U.S.A. 94, 4853-4860 (1997).

9. K. C. Neuman, T. Lionnet, and J. F. Allemand, "Single-molecule micromanipulation techniques," Аппи. Rev. Mater. Res. 37, 33-67 (2007).

10. A. E. Cohen, "Control of nanoparticles with arbitrary twodimensional force fields," Phys. Rev. Lett. 94, 118102 (2005).

11. A. E. Cohen, "Trapping and manipulating single molecules in solution," Ph.D. Dissertation, Stanford University (2006).

12. A. E. Cohen and W. E. Mourner, "Controlling Brownian motion of single protein molecules and single fluorophores in aqueous buffer," Opt. Express 16, 6941-6956 (2008).

13. L. M. Davis, B. K. Canfield, X. Li, W. H. Hofmeister, I. P. LescanoMendoza, B. W. Bomar, J. P. Wikswo, D. A. Markov, P. C. Samson, C. Daniel, Z. Sikorski, and W. Robinson, "Electrokinetic delivery of single fluorescent biomolecules in fluidic nanochannels," Proc. SPIE 7035, 70350A (2008).

14. W. E. Mourner, "New directions in single-molecule imaging and analysis," Proc. Natl. Acad. Sci. U.S.A. 104, 12596-12602 (2007).

15. W. A. Lyon and S. M. Nie, "Confinement and detection of single molecules in submicrometer channels," Anal. Chem. 69, 3400-3405 (1997).

16. J. T. Mannion and H. G. Craighead, "Nanofluidic structures for single biomolecule fluorescent detection," Biopolymers 85, 131-143 (2006).

17. L. M. Davis, Z. Sikorski, W. Robinson, G. Shen, X. Li, B. Canfield, I. Lescano, B. Bomar, W. Hofmeister, J. Germann, J. King, Y. White, and A. Terekhov, "Maximum-likelihood position sensing and actively controlled electrokinetic transport for single-molecule trapping," Proc. SPIE 6862, 68620 (2008).

18. E. B. Shera, N. K. Seitzinger, L. M. Davis, R. A. Keller, and S. A. Soper, "Detection of single fluorescent molecules," Chem. Phys. Lett. 174, 553-557 (1990).

19. D. H. Bunfield and L. M. Davis, "Monte Carlo simulation of a single molecule detection experiment," Appl. Opt. 37, 2315-2326 (1998).

20. L. M. Davis, J. G. K. Williams, and D. T. Lamb, "Computer simulation of gene detection without PCR by single molecule detection," Proc. SPIE 3570, 282-293 (1999).

21. T. Wohland, R. Rigler, and H. Vogel, "The standard deviation in fluorescence correlation spectroscopy," Biophys. J. 80, 2987-2999 (2001).

22. L. M. Davis, P. E. Williams, D. A. Ball, E. D. Matayoshi, and K. M. Swift, "Data reduction methods for application of fluorescence correlation spectroscopy to pharmaceutical drug discovery," Curr. Pharm. Biotechnol. 4, 451-462 (2003); 5, 481-481 (2004).

23. L. M. Davis and G. Q. Shen, "Accounting for triplet and saturation effects in fluorescence correlation spectroscopy measurements," Curn. Pharm. Biotechnol. 7, 287-301 (2006).

24. M. T. Tyn and T. W. Gusek, "Prediction of diffusion coefficients of proteins," Biosens. Bioelectron. 35, 327-338 (1990).

25. S. A. Soper, E. B. Shera, L. M. Davis, H. L. Nutter, and R. A. Keller, "The photophysical constants of several fluorescent dyes pertaining to ultrasensitive fluorescence spectroscopy," Photochem. Photobiol. 57, 972-977 (1993).

26. A. Spinelli, L. M. Davis, and H. Dautet, "Actively quenched single photon avalanche diode for high repetition rate time-gated photon counting," Rev. Sci. Instrum. 67, 55-61 (1996).

27. K. Fogarty and A. Van Orden, "Two-beam fluorescence crosscorrelation spectroscopy for simultaneous analysis of positive and negative ions in continuous-flow capillary electrophoresis," Anal. Chem. 75, 6634-6641 (2003).

28. P. Song, L. M. Davis, and G. R. Bashford, "Single molecule diffusion coefficient estimation by image analysis of simulated CCD images to aid high-throughput screening," in Proc. 31st Annual International Conference of the IEEE Engineering in Medicine and Biology Society, pp. 1396-1399 (2009). 